\title{
Higher likelihood of success in pregnancy in patients with high serum basal anti-müllerian hormone concentrations: An age-stratified analysis of 4516 women undergoing in vitro fertilization
}

\author{
Hong Ye*, Xiaodong Zhang, and Guoning Huang \\ Institute of Genetics and reproduction, Maternal and Child Health Care Hospital of Chongqing, Chongqing, China
}

\begin{abstract}
The aim of this study was to evaluate whether anti-Müllerian hormone (AMH) was a predictor of pregnancy rate after ovarian stimulation for in vitro fertilization (IVF). A total of 4516 patients were included in this retrospective analysis: 3535 patients for fresh embryo transfer and 981 for frozen embryo transfer after at least 3 months of whole embryo vitrification. Blood samples of all patients were taken on days 3-5 of their menstrual cycle for testing AMH in serum by using enzyme-linked immunosorbent assays. Four age groups ( $<35,35-37,38-40$ and $>40$ years) were divided into AMH subgroups according to their serum AMH levels (undetectable, $0.06-0.49,0.5-0.99,1-1.49,1.5-1.99,2-2.99,3-3.99,4-4.99$, and $\geq 5 \mathrm{ng} / \mathrm{mL}$ ). We found that serum AMH levels were positively correlated with the number of retrieved oocytes, fertilized oocytes, developed embryos and clinical pregnancy rate among the four age groups. In conclusion, this study demonstrates that women with high AMH levels had a higher probability of pregnancy across all age groups. However, due to our retrospective design, future randomized controlled trials are needed to corroborate these results
\end{abstract}

\section{Introduction}

The reproductive lifespan is related to the number and quality of oocytes. The depletion of the primordial follicle pool is associated with the decline of ovarian reserve. Although the number and quality of oocytes decrease with age, fertility varies among women of the same age. Consequently, a large number of measurements are available to evaluate the functional ovarian reserve, disclosing a prognostic value for ovarian response and helpful to decide the appropriate stimulation protocol of IVF procedures. Although reliable markers of the oocyte quality are not yet developed, Anti-Müllerian hormone (AMH) has been shown to represent a good marker of ovarian reserve.

The AMH, discovered in 1947 by Alfred Jost, is a homodimeric glycoprotein whose gene is located on the chromosome 19p13.3.and belongs to the transforming growth factor-beta superfamily that is produced by the granulosa cells surrounding pre-antral and small antral follicles, of independently of follicle-stimulating hormone (FSH) stimulation [1]. AMH is secreted into the circulation from the time of birth until menopause with only minimal fluctuations during the menstrual cycle. The serum level of AMH has been shown to correlate with the number of small follicles. Previous studies have demonstrated that $\mathrm{AMH}$ could reliably predict the quality of oocytes in in vitro fertilization (IVF) [2], the extremes of ovarian response to stimulation [3], and outcomes of IVF [4], although the predictive role of AMH may be only indirect and related to quantitative ovarian response that has achieved after the gonadotropin stimulation.

The predictive value of $\mathrm{AMH}$ from published evidence, the objectivity and potential standardization of AMH levels, and the convenience of testing at any time throughout the menstrual cycle lean toward AMH levels becoming the gold standard biomarker to evaluate ovarian reserve and predict ovarian response to stimulation [5]. Nevertheless, the ability of AMH to predict implantation rate, pregnancy rate, ongoing pregnancy rate, and live birth rate is poor [6,7], and the observational studies that have examined the association of AMH and outcomes of IVF have either been small [8-10] or restricted to specific subpopulations of infertility patients [11-12].

The aim of this study was to provide a large-sample observational study to determine the accuracy of AMH's predictive value with respect to pregnancy rate in the population after ovarian stimulation for IVF.

\section{Materials and methods}

\section{Study population}

The present study included data from 4516 women undergoing an IVF protocol with ovarian stimulation at IVF center of Maternal and Child Health Care Hospital of Chongqing between January 2012 and

Correspondence to: Institute of Genetics and reproduction, Maternal and Child Health Care Hospital of Chongqing, Chongqing 400013, China; E-mail: yehong1210@163.com

Dr. Hong Ye has been the medical director of Institute of Genetics and reproduction, Maternal and Child Health Care hospital of Chongqing since 2001. She engaged in ART clinical research from 1996 and set up the first IVF center in west China. She current interests mainly include reproductive endocrinology and ovarian stimulation.

Received: August 16, 2016; Accepted: September 19, 2016; Published: September 22, 2016 
Ye H (2016) Higher likelihood of success in pregnancy in patients with high serum basal anti-müllerian hormone concentrations: An age-stratified analysis of 4516 women undergoing in vitro fertilization

February 2014. The patients were recruited according to the following inclusion criteria: (1) serum basal AMH assay within 3 months before ovarian stimulation, (2) first attempted cycle for ovarian stimulation and IVF/intracytoplasmic sperm injection (ICSI), (3) first attempted embryo transfer regardless fresh or frozen embryos transfer, and (4) embryos transfer on day 3. The exclusion criteria were: (1) received hormonal therapy in the previous 3 months, (2) any genetic diseases (3) polycystic ovarian syndrome (PCOS) or endometriosis, (4) azoospermia with male partner and (5) a history of endocrine disorders. All patients signed an informed consent form approved by the Ethics Review Board of our Hospital, allowing retrieval of clinical data from patients' records in accordance with Good Clinical Practice guidelines.

\section{AMH assays}

The serum AMH concentration was measured using an enzyme-linked immunosorbent assay (ELISA) kit, according to the manufacturer's instructions (AMH ELISA, AnshLabs, UK). The minimal detectable concentration for AMH was $0.06 \mathrm{ng} / \mathrm{mL}$. The serum AMH level $<0.06 \mathrm{ng} / \mathrm{mL}$ was defined undetectable value. Before treatment, blood samples of all patients were taken for testing $\mathrm{AMH}$ in serum. Blood was drawn on days 3-5 of the menstrual cycles. Specimens were frozen within $24 \mathrm{~h}$ and stored at $-20^{\circ} \mathrm{C}$ until analysis was performed within 1 week.

\section{Ovarian stimulation protocols}

Different ovarian stimulation protocols were used, including long GnRH agonist, short GnRH agonist and mild stimulation protocols. Stimulation protocol was decided according to individual patient characteristics. Gonadotropins were prescribed at the discretion of the physicians according to the ovarian reserve tests $(\mathrm{AMH}$, antral follicular count AFC, bFSH) and age. The dosage was then adjusted according to follicular growth until the day of hCG administration.

For the long protocol, as described in our previous publication [13]. Briefly, after down-regulation with a GnRH agonist (Triptorelin Acetate, Ipsen Pharma, France), the ovaries were stimulated with gonadotropins. There were 4231 patients included in the long protocol.

For the short protocol, daily GnRH agonist (Triptorelin Acetate, Ipsen Pharma, France) and gonadotropins stimulation were started on day 2 of the menstrual cycle. There were 214 patients included in the short protocol.

For the mild stimulation protocol, Clomiphene Citrate (CC) 50 or $100 \mathrm{mg} / \mathrm{d}$ was started on day 3 of the menstrual cycle until the follicular maturation. Gonadotropins were started when dominant follicles size less than $14 \mathrm{~mm}$ after CC was given 5 days. There were 71 patients included in the mild stimulation protocol.

Human chorionic gonadotropin (HCG) (Ovidrel, Merck Serono, Switzerland) was administered when at least three follicles measured diameter $>18 \mathrm{~mm}$. Transvaginal oocyte retrieval was performed $36 \mathrm{~h}$ after HCG injection and embryo transfer was performed on day 3 after oocyte retrieval.

\section{Embryo culture}

Cumulus-enclosed oocytes were collected for insemination in $2.5 \mathrm{~mL}$ of IVF medium (G-IVF, Vitrolife Sweden AB, Sweden) and incubated in $5 \% \mathrm{O}_{2}$ and $6 \% \mathrm{CO}_{2}$ in compressed air at $37^{\circ} \mathrm{C}$ in a humidified incubator. Insemination (ICSI or IVF) and embryo culture were performed as previously described $[14,15]$. Cleavage-stage embryo assessment was conducted according to the criteria of Dale
[16] and embryos graded 1-3 were deemed developed embryos; these were transferred or vitrified on day 3 post-insemination following doctor's advice or patient's request. Embryo culture was carried out in $50 \mu \mathrm{L}$ micro-drops ( 1 normal fertilized embryo per drop) at $37^{\circ} \mathrm{C}$ in an incubator with humidified atmosphere of $5 \% \mathrm{O}_{2}$ and $6 \% \mathrm{CO}_{2}$ in compressed air.

\section{Vitrification and warming}

Embryos from patients who cancelled their transfers because of potential ovarian hyperstimulation syndrome (OHSS), improper endometrial preparation, or personal reasons, as well as surplus embryos after transfer, were vitrified for cryopreservation. All of embryos were frozen at day 2 or 3 . First, we equilibrated embryos in VS1 solution for $5 \mathrm{~min}$ before exposure to the vitrification solution (VS 2). The vitrification method followed the instructions from commercial kits (Kita, Toyota, Japan). After placing embryos on a Cryotop sheet, excess vitrification solution was removed by aspiration with a pipette before the carrier was plunged into $\mathrm{LN}_{2}$.

A Dewar flask of $\mathrm{LN}_{2}$ containing the carriers was placed close to the microscope. Forceps were used to grasp the straw in the $\mathrm{LN}_{2}$ and place it into a dish containing $3 \mathrm{~mL}$ of $1.0 \mathrm{~mol} / \mathrm{L}$ sucrose at $37^{\circ} \mathrm{C}$ for $1 \mathrm{~min}$. Embryos were transferred sequentially to 0.5 and $0.25 \mathrm{~mol} / \mathrm{L}$ sucrose solutions at room temperature for $3 \mathrm{~min}$ each. The embryos were then washed several times in Quinn's 1024 (Cooper Surgical, CT, USA) solution and placed in G1 medium (Vitrolife, Kungsbacka, Sweden) for further culture. Post-thaw survival of cryopreserved embryos was defined as survival with more than half of the original cells intact.

\section{Endometrial preparation of artificial cycle for frozen embryo transfer (FET)}

On day 3 of the menstrual cycle, patients commenced oral Estradiol Valeratee (Progynova, Bayer, Germany) at a dosage of 4-8 mg/d. After $14 \mathrm{~d}$ of estradiol preparation, an ultrasound was performed. If no dominant follicle was present, and the endometrial thickness was $\geq 8 \mathrm{~mm}$, progesterone $100 \mathrm{mg} / \mathrm{d}$ intramuscular injection ( $\mathrm{Li} \mathrm{Zhu}$, China) was added to the regime, and cleavage embryos were thawed on day 3 after progesterone administered and transferred on day 4 after progesterone administered.

\section{Embryo transfer}

Laser-assisted hatching [16,17] by zona drilling was performed on transferred embryos in all cases immediately before the embryo transfer. The embryo transfers were all performed with ultrasonographic guidance. Patients received luteal support (combination of estrogen and progesterone) until the blood HCG test day (14 d after embryo transfer). A clinical pregnancy was established when the gestational sac was detected by transvaginal ultrasound $28 \mathrm{~d}$ after embryo transfer.

\section{Outcome measurements and age-group stratification defined}

In determining clinical pregnancy, the denominator was the number of patients who underwent embryo transfer. In determining the developed embryo rate, the denominator was the number of cleaved $2 \mathrm{PN}$ zygotes. In determining fertilization rate, the denominator was the number of oocytes used for insemination. In determining metaphase II (MII) oocyte rate, the denominator was the number of oocytes obtained. The MII oocytes were decided at cumulus cell removal after 2-4 h of egg insemination in conventional IVF or at ICSI. The insemination with early cumulus cells removal made the MII oocyte rate measurement more objective than overnight insemination. The age stratification was 
Ye H (2016) Higher likelihood of success in pregnancy in patients with high serum basal anti-müllerian hormone concentrations: An age-stratified analysis of 4516 women undergoing in vitro fertilization

classified according to the 2013 Assisted Reproductive Technology National Summary Report published by the U.S. Centers for Disease Control and Prevention (CDC, 2013) [18].

\section{Statistical analyses}

Continuous data are presented as mean \pm sd and $25^{\text {th }}, 50^{\text {th }}$, and $75^{\text {th }}$ percentiles. One-way ANOVA with Tukey's test for multiple comparisons and Chi-square test were performed in different age groups for patient characteristics and treatment outcomes of each AMH subgroup. Proportions of pregnancy rates were compared using trend Chi-square tests. A two-tailed value of $\mathrm{P}<0.05$ was considered statistically significant.

\section{Results}

A total of 4516 patients were included in this retrospective analysis (Figure 1); 3535 underwent their first fresh embryo transfer, and 981 underwent their first frozen embryo transfer after at least 3 months of whole embryo vitrification. The mean age of the 4516 patients who underwent oocyte retrieval was $31.2 \pm 4.63$ years. Their mean serum AMH level was $3.88 \pm 3.18 \mathrm{ng} / \mathrm{ml}$, BMI was $22.07 \pm 2.69 \mathrm{~kg} / \mathrm{m}^{2}$ and oocyte number was $11.43 \pm 6.07$ (Table 1 ).

With respect to analysis stratified by age $(<35,35-37,38-40$ and $>40$ years) in the subgroups of various baseline serum AMH

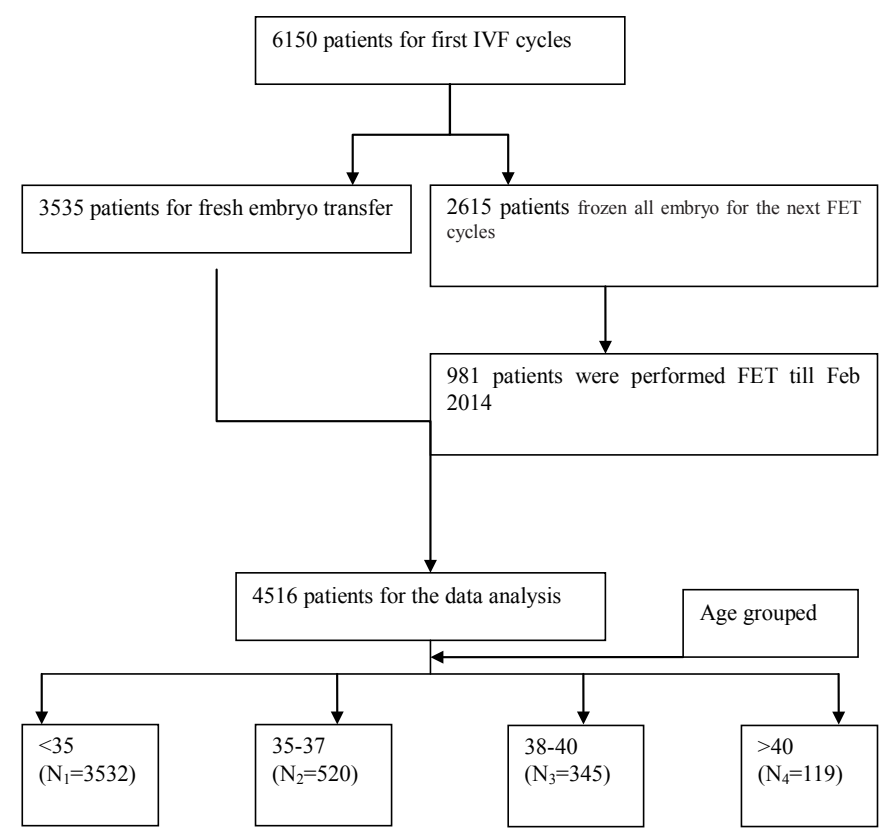

Figure 1. The flow chart of data recruitment (undetectable,0.06-0.49, 0.5-0.99, 1-1.49, 1.5-1.99, 2-2.99, 3-3.99, $4-4.99$, and $\geq 5 \mathrm{ng} / \mathrm{mL}$ ), it demonstrated the number of retrieved oocytes, fertilized oocytes and developed embryos positively correlated with baseline serum AMH in all age groups $(<35,35-37,38-40$ and $>40$ years) $(\mathrm{p}<0.05)$, and the number of matured oocytes positively correlated with baseline serum AMH in the age $<35$ group $(\mathrm{p}<0.01)$. However, the rate of mature oocytes and fertilization in all age groups did not differ according to baseline serum AMH ( $p>0.05)$. The study also demonstrated the rate of developed embryos in all age groups except age $>40$ group which negatively correlated with baseline serum AMH $(\mathrm{p}<0.05)$. For age $>40$ group, the rate of developed embryos did not differ according to baseline serum AMH ( $\mathrm{p}=0.63$ ) (Table 2-5).

The trend Chi-square tests showed that clinical pregnancy rates among age $<35,35-37$ and 38-40 groups were positively correlated with baseline serum AMH $(\mathrm{p}<0.05)$ (Table 6).

\section{Discussion}

AMH has demonstrated its effectiveness in many areas. It is the best hormonal marker of ovarian reserve, and is a useful tool to predict low response to controlled ovarian stimulation and conversely the risk of excessive response [19]. Our study showed that higher AMH was related to more retrieved oocytes independent of age, which are consistent with this previous report on ovarian reserve. High AMH expression was detected in primary, secondary, pre-antral, and early antral follicles $<4 \mathrm{~mm}$ in diameter, but this sharply decreased in follicles $8 \mathrm{~mm}$ in diameter [20,21]. Due to peripheral circulating concentrations of $\mathrm{AMH}$ being proportional to the primordial follicle pool, $\mathrm{AMH}$ may indirectly represent the ovarian reserve and ovarian response to gonadotropins stimulation.

The ability of AMH to predict pregnancy is contradictory, as a meta-analysis of individual patient data clearly demonstrated that $\mathrm{AMH}$ does not predict ongoing pregnancy in IVF [22]. However, another meta-analysis of diagnostic accuracy showed that AMH adds some value in predicting live birth, and that this is independent of age or immune assayable AMH [7]. To the best of our knowledge, this is the largest retrospective analysis investigating the relationship between serum AMH and the rate of clinical pregnancy after first IVF cycles. Our retrospective stratified analysis found that clinical pregnancy rate among age $<35,35-37$ and 38-40 groups were positively correlated with baseline serum AMH independent of age. Although several investigators have evaluated an AMH cut off value for predicting clinical pregnancy rate and live birth rate in certain women undergoing IVF $[2,4,7,10,23,24]$, only one study indicated that follicle fluid AMH was significantly correlated with clinical pregnancy rate and live birth rate [25]. Most of the previous reports regarding the use of AMH in prediction of outcomes in IVF were diagnostic trials, or where data were demonstrated to be in extracted form, raw data were omitted,

Table 1. Patient characteristics according to AMH percentile in oocyte retrievals $(n=4516)$.

\begin{tabular}{|c|c|c|c|c|c|c|}
\hline & Mean \pm sd & Minimum & $25^{\text {th }}$ percentile & $5^{\text {th }}$ percentile & $75^{\text {th }}$ percentile & Maximum \\
\hline Age (years) & $31.2 \pm 4.64$ & 20 & 28 & 31 & 34 & 48 \\
\hline $\mathrm{AMH}(\mathrm{ng} / \mathrm{mL})$ & $3.876 \pm 3.179$ & undetectable & 1.63 & 3.03 & 5.17 & 28.12 \\
\hline BMI $\left(\mathrm{kg} / \mathrm{m}^{2}\right)$ & $22.07 \pm 2.69$ & 14.98 & 19.88 & 21.48 & 23.44 & 37.33 \\
\hline No. of oocytes & $11.43 \pm 6.071$ & 1 & 7 & 11 & 15 & 37 \\
\hline No. of MII oocyte & $11.43 \pm 5.99$ & 1 & 7 & 11 & 15 & 37 \\
\hline MII rate $(\%)$ & $0.89 \pm 0.12$ & 0.38 & 0.83 & 0.92 & 1 & 1 \\
\hline No. of fertilized oocytes & $9.12 \pm 5.36$ & 1 & 5 & 9 & 13 & 35 \\
\hline Fertilization rate $(\%)$ & $0.81 \pm 0.21$ & 0.02 & 0.71 & 0.86 & 1 & 1 \\
\hline No. of developed embryos & $4.56 \pm 3.32$ & 1 & 2 & 4 & 6 & 27 \\
\hline Developed embryo rate $(\%)$ & $0.64 \pm 0.26$ & 0.01 & 0.45 & $0 . .64$ & 0.83 & 1 \\
\hline
\end{tabular}


Ye H (2016) Higher likelihood of success in pregnancy in patients with high serum basal anti-müllerian hormone concentrations: An age-stratified analysis of 4516 women undergoing in vitro fertilization

Table 2. Treatment outcomes according to baseline serum AMH in age $<35$ group $(\mathrm{n}=3532)$.

\begin{tabular}{|l|c|c|c|c|c|c|c|c|c|c|}
\hline & Undetectable & $\mathbf{0 . 0 6 - 0 . 4 9}$ & $\mathbf{0 . 5 - 0 . 9 9}$ & $\mathbf{1 - 1 . 4 9}$ & $\mathbf{1 . 5 - 1 . 9 9}$ & $\mathbf{2 - 2 . 9 9}$ & $\mathbf{3 - 3 . 9 9}$ & $\mathbf{4 - 4 . 9 9}$ & $\mathbf{2 5}$ & $\mathbf{P}$ \\
\hline No. patients & 2 & 63 & 200 & 335 & 385 & 701 & 568 & 375 & 903 & 11 \\
\hline No. oocytes & 11 & $4.43 \pm 2.72$ & $6.77 \pm 3.43$ & $8.32 \pm 3.98$ & $9.71 \pm 3.95$ & $10.82 \pm 4.68$ & $12.49 \pm 4.86$ & $13.35 \pm 4.94$ & $14.65 \pm 5.87$ & $<0.01$ \\
\hline No. MII oocytes & $10 \pm 1.41$ & $3.5 \pm 2.06$ & $5.26 \pm 2.18$ & $7.42 \pm 3.99$ & $8.66 \pm 3.91$ & $9.48 \pm 4.55$ & $11.38 \pm 4.99$ & $11.99 \pm 5.02$ & $12.11 \pm 5.77$ & $<0.01$ \\
\hline MII oocyte rate & $0.9 \pm 0.1$ & $0.875 \pm 0.14$ & $0.89 \pm 0.11$ & $0.91 \pm 0.12$ & $0.85 \pm 0.16$ & $0.89 \pm 0.12$ & $0.89 \pm 0.12$ & $0.86 \pm 0.13$ & $0.91 \pm 0.11$ & 0.073 \\
\hline No. fertilized oocytes & $7.5 \pm 0.7$ & $3.11 \pm 2.44$ & $4.86 \pm 3.08$ & $6.81 \pm 3.73$ & $7.85 \pm 3.63$ & $8.84 \pm 4.29$ & $10.25 \pm 4.41$ & $10.56 \pm 4.54$ & $11.68 \pm 5.24$ & $<0.01$ \\
\hline Fertilization rate & $0.9 \pm 0.22$ & $0.86 \pm 0.22$ & $0.83 \pm 0.28$ & $0.84 \pm 0.28$ & $0.82 \pm 0.24$ & $0.84 \pm 0.25$ & $0.85 \pm 0.24$ & $0.83 \pm 0.18$ & $0.81 \pm 0.18$ & 0.11 \\
\hline No. developed embryos & 2 & $2.11 \pm 2.44$ & $3.99 \pm 3.08$ & $4.81 \pm 3.13$ & $5.48 \pm 2.61$ & $5.84 \pm 4.29$ & $5.05 \pm 3.07$ & $5.06 \pm 3.14$ & $5.8 \pm 3.36$ & $<0.01$ \\
\hline Developed embryo rate & 0.25 & $0.76 \pm 0.27$ & $0.69 \pm 0.29$ & $0.67 \pm 0.27$ & $0.62 \pm 0.24$ & $0.6 \pm 0.25$ & $0.6 \pm 0.24$ & $0.59 \pm 0.25$ & $0.6 \pm 0.24$ & $<0.01$ \\
\hline
\end{tabular}

Table 3. Treatment outcomes according to baseline serum AMH in age35-37 group ( $\mathrm{n}=520)$.

\begin{tabular}{|c|c|c|c|c|c|c|c|c|c|c|}
\hline & Undetectable & $0.06-0.49$ & $0.5-0.99$ & $1-1.49$ & 1.5-1.99 & $2-2.99$ & $3-3.99$ & $4-4.99$ & $\geq 5$ & $\mathbf{P}$ \\
\hline No. patients & 0 & 31 & 66 & 64 & 81 & 107 & 73 & 37 & 61 & \\
\hline No. oocytes & - & $4.12 \pm 2.43$ & $6.03 \pm 3.52$ & $7.41 \pm 4.01$ & $9.28 \pm 4.11$ & $10.11 \pm 3.86$ & $11.42 \pm 4.21$ & $13.08 \pm 3.83$ & $14.08 \pm 5.52$ & $<0.01$ \\
\hline No. MII oocytes & - & $3.81 \pm 2.07$ & $5.85 \pm 1.95$ & $5.83 \pm 2.45$ & $8.41 \pm 3.98$ & $9.24 \pm 4.86$ & $11 \pm 5.66$ & $11 \pm 5.89$ & $11.57 \pm 6.33$ & 0.33 \\
\hline MII oocyte rate & - & $0.91 \pm 0.12$ & $0.88 \pm 0.11$ & $0.92 \pm 0.11$ & $0.94 \pm 0.08$ & $0.92 \pm 0.11$ & $0.93 \pm 0.11$ & $0.89 \pm 0.11$ & $0.91 \pm 0.11$ & 0.58 \\
\hline No. fertilized oocytes & - & $3.44 \pm 2.11$ & $4.83 \pm 2.64$ & $5.77 \pm 3.08$ & $7.44 \pm 3.76$ & $8.05 \pm 3.49$ & $8.85 \pm 4.04$ & $9.29 \pm 4.51$ & $11.37 \pm 4.36$ & $<0.01$ \\
\hline Fertilization rate & - & $0.86 \pm 0.18$ & $0.83 \pm 0.18$ & $0.81 \pm 0.18$ & $0.81 \pm 0.2$ & $0.80 \pm 0.19$ & $0.78 \pm 0.2$ & $0.79 \pm 0.18$ & $0.82 \pm 0.19$ & 0.72 \\
\hline No. developed embryos & - & $2.12 \pm 1.21$ & $2.81 \pm 1.72$ & $3.16 \pm 1.94$ & $3.68 \pm 2.67$ & $4.44 \pm 2.29$ & $3.93 \pm 2.39$ & $4.73 \pm 2.44$ & $5.49 \pm 2.51$ & $<0.01$ \\
\hline Developed embryo rate & - & $0.875 \pm 0.22$ & $0.76 \pm 0.25$ & $0.71 \pm 0.24$ & $0.67 \pm 0.25$ & $0.69 \pm 0.21$ & $0.58 \pm 0.23$ & $0.58 \pm 0.21$ & $0.62 \pm 0.22$ & $<0.01$ \\
\hline
\end{tabular}

Table 4. Treatment outcomes according to baseline serum AMH in age 38-40 group $(n=345)$.

\begin{tabular}{|c|c|c|c|c|c|c|c|c|c|c|}
\hline & Undetectable & $0.06-0.49$ & $0.5-0.99$ & $1-1.49$ & 1.5-1.99 & $2-2.99$ & 3-3.99 & $4-4.99$ & $\geq 5$ & $\mathbf{P}$ \\
\hline No. patients & 1 & 28 & 60 & 68 & 53 & 62 & 43 & 13 & 17 & \\
\hline No. oocytes & 13 & $4.03 \pm 2.67$ & $4.67 \pm 2.73$ & $6.39 \pm 3.21$ & $7.91 \pm 4.63$ & $9.74 \pm 4.62$ & $11.98 \pm 4.99$ & $12.15 \pm 2.88$ & $12.53 \pm 6.7$ & 0.04 \\
\hline No. MII oocytes & 10 & $3.11 \pm 2.51$ & $4.17 \pm 2.86$ & $5 \pm 1.63$ & $6.33 \pm 4.46$ & $8.88 \pm 4.07$ & $11.8 \pm 3.49$ & $9.12 \pm 4.02$ & $10.33 \pm 6.66$ & 0.88 \\
\hline MII oocyte rate & 0.76 & $0.81 \pm 0.14$ & $0.94 \pm 0.09$ & $0.86 \pm 0.12$ & $0.78 \pm 0.23$ & $0.92 \pm 0.11$ & $0.92 \pm 0.15$ & $0.81 \pm 0.15$ & $0.83 \pm 012$ & 0.66 \\
\hline No. fertilized oocytes & 7 & $3.01 \pm 2.51$ & $3.73 \pm 2.28$ & $4.55 \pm 2.88$ & $5.41 \pm 3.46$ & $7.93 \pm 4.6$ & $9.67 \pm 4.3$ & $8.38 \pm 3.2$ & $7.35 \pm 5.3$ & 0.03 \\
\hline Fertilization rate & 0.54 & $0.91 \pm 0.15$ & $0.84 \pm 0.21$ & $0.83 \pm 0.22$ & $0.81 \pm 0.21$ & $0.83 \pm 0.17$ & $0.81 \pm 0.19$ & $0.85 \pm 0.15$ & $0.71 \pm 0.3$ & 0.09 \\
\hline No. developed embryos & 2 & $2.21 \pm 1.23$ & $2.37 \pm 1.43$ & $2.69 \pm 1.37$ & $3.45 \pm 2.34$ & $3.77 \pm 2.18$ & $4.86 \pm 3.26$ & $5.23 \pm 2.59$ & $4.12 \pm 2.57$ & 0.01 \\
\hline Developed embryo rate & 1 & $0.84 \pm 0.2$ & $0.8 \pm 0.26$ & $0.74 \pm 0.26$ & $0.67 \pm 0.24$ & $0.63 \pm 0.25$ & $0.63 \pm 0.25$ & $0.62 \pm 0.24$ & $0.63 \pm 0.18$ & 0.03 \\
\hline
\end{tabular}

Table 5. Treatment outcomes according to baseline serum AMH in age $>40$ group $(n=119)$.

\begin{tabular}{|c|c|c|c|c|c|c|c|c|c|c|}
\hline & Undetectable & $0.06-0.49$ & $0.5-0.99$ & $1-1.49$ & 1.5-1.99 & $2-2.99$ & 3-3.99 & $4-4.99$ & $\geq 5$ & $\mathbf{P}$ \\
\hline No. patients & 6 & 22 & 36 & 23 & 11 & 16 & 1 & 2 & 2 & \\
\hline No. oocytes & $1.5 \pm 0.55$ & $2.3 \pm 1.03$ & $3.13 \pm 1.89$ & $5.09 \pm 3.19$ & $5.22 \pm 2.28$ & $7.21 \pm 4.39$ & 9 & $9.5 \pm 6.36$ & $17 \pm 2.82$ & 0.04 \\
\hline No. MII oocytes & $1.4 \pm 0.6$ & $2.21 \pm 2.12$ & $2.33 \pm 2.25$ & $4.25 \pm 2.1$ & $4.5 \pm 3.53$ & $6.5 \pm 0.707$ & 9 & 9 & 16 & 0.91 \\
\hline MII oocyte rate & $0.9 \pm 0.2$ & $0.88 \pm 0.19$ & $0.86 \pm 0.22$ & $0.88 \pm 0.21$ & $0.87 \pm 0.05$ & $0.85 \pm 0.01$ & 1 & $0.9 \pm 0.14$ & $0.9 \pm 0.14$ & 0.82 \\
\hline No. fertilized oocytes & $1.3 \pm 0.55$ & $1.76 \pm 0.84$ & $2.07 \pm 1.43$ & $4.04 \pm 2.91$ & $3.74 \pm 2.84$ & $4.19 \pm 3.17$ & 8 & $8.5 \pm 7.07$ & $13 \pm 1.41$ & 0.04 \\
\hline Fertilization rate & $0.9 \pm 0.15$ & $0.81 \pm 0.26$ & $0.86 \pm 0.19$ & $0.91 \pm 0.14$ & $0.75 \pm 0.21$ & $0.79 \pm 0.24$ & 0.89 & $0.9 \pm 0.14$ & $0.77 \pm 0.04$ & 0.44 \\
\hline No. developed embryos & $1.2 \pm 0.5$ & $1.43 \pm 0.55$ & $1.86 \pm 0.75$ & $2.96 \pm 1.02$ & $2.82 \pm 1.99$ & $2.87 \pm 1.4$ & 5 & $5 \pm 5.66$ & 2 & 0.05 \\
\hline Developed embryo rate & $0.9 \pm 0.22$ & $0.91 \pm 0.19$ & $0.79 \pm 0.29$ & $0.82 \pm 0.21$ & $0.78 \pm 0.19$ & $0.73 \pm 0.23$ & 0.71 & $0.9 \pm 0.13$ & $0.42 \pm 0.35$ & 0.63 \\
\hline
\end{tabular}

Table 6. Clinical pregnancy rate according to baseline serum AMH in patients stratified by age.

\begin{tabular}{|c|c|c|c|c|c|c|c|c|c|c|}
\hline Age & Undetectable & 0.06-0.49 & $0.5-0.99$ & $1-1.49$ & $1.5-1.99$ & $2-2.99$ & $3-3.99$ & $4-4.99$ & $\geq 5$ & Trend $P$ \\
\hline$<35$ & $\begin{array}{l}100 \% \\
(2 / 2)\end{array}$ & $\begin{array}{l}49.2 \% \\
(31 / 63)\end{array}$ & $\begin{array}{c}58.03 \% \\
(112 / 193)\end{array}$ & $\begin{array}{c}58.21 \% \\
(195 / 335)\end{array}$ & $\begin{array}{c}56.36 \% \\
(217 / 385)\end{array}$ & $\begin{array}{c}60.63 \% \\
(425 / 701)\end{array}$ & $\begin{array}{c}60.74 \% \\
(345 / 568)\end{array}$ & $\begin{array}{c}66.40 \% \\
(249 / 375)\end{array}$ & $\begin{array}{c}63.96 \% \\
(582 / 910)\end{array}$ & 0.002 \\
\hline $35-37$ & - & $\begin{array}{l}53.13 \% \\
(17 / 32)\end{array}$ & $\begin{array}{l}42.42 \% \\
(28 / 66)\end{array}$ & $\begin{array}{l}53.13 \% \\
(34 / 64)\end{array}$ & $\begin{array}{l}45.58 \% \\
(37 / 81)\end{array}$ & $\begin{array}{l}49.53 \% \\
(53 / 107)\end{array}$ & $\begin{array}{l}39.73 \% \\
(29 / 73)\end{array}$ & $\begin{array}{l}56.76 \% \\
(21 / 37)\end{array}$ & $\begin{array}{c}65 \% \\
(39 / 60)\end{array}$ & 0.000 \\
\hline $38-40$ & $\overline{0}$ & $\begin{array}{l}35.71 \% \\
(10 / 28)\end{array}$ & $\begin{array}{c}30 \% \\
(18 / 60)\end{array}$ & $\begin{array}{l}39.13 \% \\
(27 / 69)\end{array}$ & $\begin{array}{l}30.19 \% \\
(16 / 53)\end{array}$ & $\begin{array}{l}45.16 \% \\
(28 / 62)\end{array}$ & $\begin{array}{l}41.86 \% \\
(18 / 43)\end{array}$ & $\begin{array}{c}23.07 \% \\
(3 / 13)\end{array}$ & $\begin{array}{l}58.88 \% \\
(10 / 17)\end{array}$ & 0.000 \\
\hline$>40$ & $\begin{array}{c}- \\
(0 / 6)\end{array}$ & $\begin{array}{c}- \\
(0 / 21)\end{array}$ & $\begin{array}{c}25 \% \\
(9 / 36)\end{array}$ & $\begin{array}{l}26.08 \% \\
(6 / 23)\end{array}$ & $\begin{array}{c}14.29 \% \\
(1 / 7)\end{array}$ & $\begin{array}{c}25 \% \\
(4 / 16)\end{array}$ & $\begin{array}{c}- \\
(0 / 1)\end{array}$ & $\begin{array}{c}33.33 \% \\
(1 / 3)\end{array}$ & $\begin{array}{c}- \\
(0 / 6)\end{array}$ & - \\
\hline
\end{tabular}

The AMH undetectable subgroup and the age $>40$ groups were not calculated for the trend analysis, because the numerators were zero.

or data could only provide limited cues toward further randomized controlled studies $[2,4,7,10,23,24]$. Although inherent drawbacks exist in the present study due to the retrospective design, our large sample size and specialized infertility distribution made this analysis less biased. In addition, a narrowing of groups where AMH was less than $5 \mathrm{ng} / \mathrm{mL}$ made the results more transparent than those from previous diagnostic trials. 
Ye H (2016) Higher likelihood of success in pregnancy in patients with high serum basal anti-müllerian hormone concentrations: An age-stratified analysis of 4516 women undergoing in vitro fertilization

With respect to zygotes and embryos, our study showed that High $\mathrm{AMH}$ levels were correlated with high absolute numbers of zygotes, and developed embryos, but augmented rates were not observed; to the contrary, the rate of developed embryo in all age groups except age $>40$ group which negatively correlated with baseline serum AMH. This might be due to subjective judgments by the embryologist, especially with patients who delivered few oocytes compared with patients where an abundant number of oocytes were retrieved, and normal fertilization (2PN)-derived embryos appear especially important. Although embryonic selection criteria were objective, the embryologist may show bias in judgment, and a cumulative effect due to large sample size may alter the trend in developed embryo rate; this result differs from previous reports $[4,24,25]$.

In conclusion, we demonstrate in the present study that women with high AMH levels have a higher probability of pregnancy across all age groups. However, due to the retrospective experimental design, future RCT studies are needed to corroborate these results.

\section{References}

1. Majumder K, Gelbaya TA, Laing I, Nardo LG (2010) The use of anti-Müllerian hormone and antral follicle count to predict the potential of oocytes and embryos. Eur J Obstet Gynecol Reprod Biol 150: 166-170.[Crossref]

2. Lehmann P, Vélez MP, Saumet J, Lapensée L, Jamal W, et al. (2014) Anti-Müllerian hormone (AMH): a reliable biomarker of oocyte quality in IVF. J Assist Reprod Genet 31: 493-498.[Crossref]

3. Nardo LG, Gelbaya TA, Wilkinson H, Roberts SA, Yates A, et al. (2009) Circulating basal anti-Müllerian hormone levels as predictor of ovarian response in women undergoing ovarian stimulation for in vitro fertilization. Fertil Steril 92: 1586-1593. [Crossref]

4. Brodin T, Hadziosmanovic N, Berglund L, Olovsson M, Holte J (2013) Antimüllerian hormone levels are strongly associated with live-birth rates after assisted reproduction. J Clin Endocrinol Metab 98: 1107-1114.[Crossref]

5. Practice Committee of the American Society for Reproductive Medicine (2015) Testing and interpreting measures of ovarian reserve: a committee opinion. FertilSteril103: e99e17.[Crossref]

6. Tal R, Tal O, Seifer BJ, Seifer DB (2015) Antimüllerian hormone as predictor ofimplantation and clinical pregnancy after assisted conception: a systematicreview and meta-analysis. Fertil Steril 103:119-130. [Crossref]

7. Iliodromiti S, Kelsey TW, Wu O, Anderson RA, Nelson SM (2014) The predictive accuracy of anti-Müllerian hormone for live birth after assisted conception: a systematic review and meta-analysis of the literature. Hum Reprod Update 20: 560-570.[Crossref]

8. Nelson SM, Yates RW, Fleming R (2007) Serum anti-Müllerian hormone and FSH prediction of live birth and extremes of response in stimulated cycles--implications for individualization of therapy. Hum Reprod 22: 2414-2421.[Crossref]

9. Lee TH, Liu CH, Huang CC, Hsieh KC, Lin PM, et al. (2009) Impact of female age and male infertility on ovarian reserve markers to predict outcome of assisted reproduction technology cycles. Reprod Biol Endocrinol 7:100. [Crossref]

10. Gleicher N, Weghofer A, Barad DH (2010) Anti-Müllerian hormone (AMH) defines, independent of age, low versus good live-birth chances in women with severely diminished ovarian reserve. Fertil Steril 94: 2824-2827.[Crossref]

11. Arce JC, La Marca A, Mirner Klein B, Nyboe Andersen A, Fleming R (2013) Antimüllerian hormone in gonadotropin releasing-hormone antagonist cycles: prediction of ovarian response and cumulative treatment outcome in good-prognosis patients. Fertil Steril 99: 1644-1653.[Crossref]

12. Ye H, Huang G-N, Zeng P-H, Pei L (2009) IVF/ICSI outcomes between cycles with luteal estradiol (E2) pre-treatment before GnRH antagonist protocol and standard long GnRH agonist protocol: a prospective and randomized study. J Assist Reprod Genet 26:105-111. [Crossref]

13. Xiong S, Han W, Liu JX, Zhang XD, Liu WW, et al. (2011) Effects of cumulus cells removal after $6 \mathrm{~h}$ co-incubation of gametes on the outcomes of human IVF. $J$ Assist Reprod Genet 28: 1205-1211.[Crossref]

14. Zhang XD, Liu JX, Liu WW, Gao Y, Han W, et al. (2013) Time of insemination culture and outcomes of in vitro fertilization: a systematic review and meta-analysis. Hum Reprod Update 19: 685-695.[Crossref]

15. Dale B, Elder K. In vitro Fertilization. United Kingdom: Cambrige University; 1997 pp. 115-6.

16. Grace J, Bolton V, Braude P, Khalaf Y (2007) Assisted hatching is more effective when embryo quality was optimal in previous failed IVF/ICSI cycles. J Obstet Gynaecol 27: 56-60.[Crossref]

17. Sallam HN, Sadek SS, Agameya AF (2003) Assisted hatching--a meta-analysis of randomized controlled trials. $J$ Assist Reprod Genet 20: 332-342.[Crossref]

18. Assisted reproductive technology national summary report (2013) http://www.cdc.gov/ art/reports/2013/national-summary.html

19. La Marca A, Sighinolfi G, Radi D, Argento C, Baraldi E, et al. (2010) Anti-Mullerian hormone $(\mathrm{AMH})$ as a predictive marker in assisted reproductive technology (ART) Hum Reprod Update 16: 113-130.[Crossref]

20. Weenen C, Laven JS, Von Bergh AR, Cranfield M, Groome NP, et al. (2004) AntiMullerian hormone expression pattern in thehuman ovary: potentialimplications for initial and cyclic follicle recruitment. Mol Hum Reprod 10: 77-83. [Crossref]

21. Jeppesen JV, Anderson RA, KelseyTW,Christiansen SL, Kristensen SG, et al. (2013) Which follicles make the mostanti-Mullerian hormone in humans? Evidence for an abrupt decline in AMHproduction at the time of follicle selection. Mol Hum Reprod 19:519-527. [Crossref]

22. Broer SL, van DJ, Broeze KA, Dolleman M, Opmeer BC, et al. (2013) Added value of ovarian reserve testing on patientcharacteristics in the prediction of ovarian response and ongoing pregnancy: anindividual patient data approach. Hum Reprod Update 19: 26-36. [Crossref]

23. Koshy AK, Gudi A, Shah A, Bhide P, Timms P, et al. (2013) Pregnancy prognosis in women with anti-Müllerian hormone below the tenth percentile. Gynecol Endocrinol 29: 662-665.[Crossref]

24. Kim JH, Lee JR, Chang HJ, Jee BC, Suh CS, et al. (2014) Anti-Müllerian hormone levels in the follicular fluid of the preovulatory follicle: a predictor for oocyte fertilization and quality of embryo. J Korean Med Sci29: 1266-1270.[Crossref]

25. Lin WQ, Yao LN, Zhang DX, Zhang W, Yang XJ, et al. (2013) The predictive value ofanti-Muellerian hormone on embryo quality, blastocyst development, and pregnancyrate following in vitro fertilization-embryo transfer (IVF-ET). $J$ Assist Reprod Genet 30: 649-655.[Crossref]

Copyright: (C2016 Ye H. This is an open-access article distributed under the terms of the Creative Commons Attribution License, which permits unrestricted use, distribution, and reproduction in any medium, provided the original author and source are credited. 